\title{
Parallel imaging acceleration of spiral Fourier velocity encoded MRI using SPIRiT
}

\author{
Davi M. Lyra-Leite and Joao L. A. Carvalho
}

\begin{abstract}
This paper demonstrates parallel imaging acceleration of spiral Fourier velocity encoded MRI using the iterative self-consistent parallel imaging reconstruction (SPIRiT) technique. Magnitude images and time-velocity distributions obtained with image domain SPIRiT and sum-of-squares reconstruction are compared, for 2-fold and 4-fold undersampling. We show that SPIRiT is able to reduce spatial aliasing from undersampled time-velocity distributions, with good results for 2fold undersampling, and moderately good results for 4-fold undersampling.
\end{abstract}

\section{INTRODUCTION}

Magnetic resonance imaging (MRI) of living human tissue started in the 1970s. Due to its recentness, MRI is a very fruitful area of research in the bioengineering and signal processing fields [1]. MRI allows the study of both body anatomy and function. New developments in flow imaging and flow velocity distribution assessment have improved the precision of MRI flow assessment techniques.

Fourier velocity encoding (FVE) [2] is useful in the assessment of valvular disease [3], [4], as it eliminates partialvolume effects that may cause loss of diagnostic information in phase-contrast imaging [5]. FVE has also been proposed as a method for measuring wall shear rate in the carotid arteries [6].

Although the scan-time of 2DFT FVE is prohibitively long for clinical use, the spiral FVE method [3] shows promise, as it is substantially faster. The scan time in FVE can be significantly reduced using temporal acceleration [7].

The temporal resolution of temporally-accelerated spiral FVE can be improved if spatial aliasing due to temporal undersampling is reduced. This may be achieved using parallel imaging. We investigate the use of the iterative selfconsistent parallel imaging reconstruction (SPIRiT) method [8] to accelerate the acquisition of spiral FVE data.

\section{A. Spiral FVE}

Spiral Fourier velocity encoding is a magnetic resonance imaging technique for assessment of blood flow in human vessels. The pulse sequence consists of slice-selective excitation, a velocity-encoding bipolar gradient along $z$, a $4 \mathrm{~ms}$ spiral readout, and spoiler and refocusing gradients [3]. The acquired data consist of a temporally-resolved stack-ofspirals in $k_{x}-k_{y}-k_{v}$ space [3]. A Cartesian inverse Fourier transform along $k_{v}$, followed by a non-Cartesian inverse

\footnotetext{
*This work was supported by the Brazilian National Council for Technological and Scientific Development $(\mathrm{CNPq})$.

D. M. Lyra-Leite and J. L. A. Carvalho are with the Department of Electrical Engineering, University of Brasilia, Brasilia, DF, Brazil davi at ieee.org and joaoluiz at pgea.unb.br
}

Fourier transform [9] along $k_{x}-k_{y}$, produces the spatiotemporal-velocity distribution, $m(x, y, v, t)$.

\section{B. SPIRiT}

The iterative self-consistent parallel imaging reconstruction (SPIRiT) approach [8] is an autocalibrated coil-bycoil parallel imaging reconstruction method, based on selfconsistency. In this work, we used image domain SPIRiT, because the data was acquired in a non-Cartesian fashion (spiral trajectory in k-space).

The main idea of this technique is to use information from all neighborhood points in all coils - sampled or not - in order to obtain the missing data. Using this parallel imaging reconstruction algorithm, it is possible to recover the missing information from undersampled data.

\section{Methods}

Spiral FVE scans were performed on a GE Signa 3T EXCITE HD system $(40 \mathrm{mT} / \mathrm{m}$ maximum gradient amplitude, $150 \mathrm{~T} / \mathrm{m} / \mathrm{s}$ maximum gradient slew rate), using a 4channel carotid coil. Scan parameters were: $1.4 \times 1.4 \times 5 \mathrm{~mm}^{3}$ spatial resolution over a $16 \mathrm{~cm}$ field-of-view, $5 \mathrm{~cm} / \mathrm{s}$ velocity resolution over a $240 \mathrm{~cm} / \mathrm{s}$ field-of-view, eight $4 \mathrm{~ms}$ variabledensity spiral readouts per temporal frame and $k_{v}$ coordinate, and $12 \mathrm{~ms}$ temporal resolution. Scan time was 146 seconds (256 heartbeats at $105 \mathrm{bpm}$ ). The institutional review board of the University of Southern California approved the imaging protocols. Subjects were screened for magnetic resonance imaging risk factors and provided informed consent in accordance with institutional policy.

Parallel imaging acceleration was evaluated using 2-fold and 4-fold spatially-undersampled datasets, obtained from the fully-sampled spiral FVE dataset. The undersampled data was reconstructed in MATLAB using two techniques: sumof-squares (SoS) [10] — which simply combines the images obtained from the undersampled data, without reducing aliasing - , and image-domain SPIRiT [8].

The voxels of interest, corresponding to the major blood vessels in the neck, were manually selected on an axial image obtained from the fully-sampled data. The studied vessels were: right internal carotid artery (RICA), right external carotid artery (RECA), right jugular vein (RJV), left jugular vein (LJV), left carotid bifurcation (LCB), right vertebral artery (RVA), and left vertebral artery (LVA). The spatial coordinates of these voxels were used for obtaining the timevelocity distributions associated with each of these vessels.

Magnitude images corresponding to an axial view of the neck, and the time-velocity distributions associated with each 
blood vessel, were reconstructed using both SoS and SPIRiT techniques and the results were compared quantitatively (signal-to-error ratio - SER — , relative to the fully-sampled SoS result) and qualitatively (by visual inspection).

\section{RESUlTS}

SoS reconstruction displays increased signal at $v=0 \mathrm{~cm} / \mathrm{s}$, at both 2-fold and 4-fold undersampling (Figures 1, 2 and 3). This is because the majority of the aliasing signal comes from static tissue. SPIRiT is able to reduce spatial aliasing from undersampled spiral FVE, with qualitatively good results at 2-fold undersampling, in both spatial (Figure 1) and time-velocity domains (Figures 2 and 3), and moderately good results at 4-fold undersampling.

The results in Figure 1 show that the spatial images obtained with SPIRiT reconstruction for both 2-fold and 4fold undersampled datasets clearly present the main features of the neck. In these two images, it is possible to identify the arteries and veins, even though some aliasing remains in the 4-fold undersampled image. In comparison, it is almost impossible to clearly identify the RICA, RECA, and other important blood vessels from the 4-fold undersampled image reconstructed using sum-of-squares. Note that the spatial image resolution is low in all images (including the fullysampled image), because (i) spiral FVE is robust to partial voluming, so it does not require high spatial resolution for accurate flow assessment; (ii) the spatial resolution was penalized in order to achieve high velocity and temporal resolutions in a short scan; and (iii) off-resonance effects at $3 \mathrm{~T}$ are twice as stronger as in $1.5 \mathrm{~T}$, and cause spatial blurring in spiral imaging (we used short readouts in order to reduce this effect).

The velocity-distribution results presented in Figures 2 and 3 show that 2-fold accelerated SPIRiT was able to significantly remove the aliasing signal at $v=0 \mathrm{~cm} / \mathrm{s}$ that is observed with SoS reconstruction. This is an important result, as we intend to use SPIRiT to reduce spatial aliasing and hence allow for improved temporal resolution of temporally-accelerated spiral FVE [7]. However, SPIRiT reconstruction introduces artifacts on the flow curves, which is an undesirable result. Such artifacts are not observed in the 2-fold undersampled SoS results, where aliasing artifacts are present only at $v=0 \mathrm{~cm} / \mathrm{s}$, as discussed above. At 4-fold undersampling, SoS displays even stronger aliasing artifacts at $v=0 \mathrm{~cm} / \mathrm{s}$, and also some artifacts on the flow curves; SPIRiT reconstruction reduces the artifacts at $v=0 \mathrm{~cm} / \mathrm{s}$, but introduces stronger artifacts on the flow curves.

A quantitative evaluation is presented in Table 1. For the magnitude spatial image for $v=0 \mathrm{~cm} / \mathrm{s}$ and $t=0 \mathrm{~s}$ (shown in Figure 1), SPIRiT shows an increase in signal-to-error ratio (SER) of over $10 \mathrm{~dB}$ relative to $\mathrm{SoS}$ reconstruction, for both 2-fold and 4-fold undersampled datasets. When looking at the time-velocity distribution results for 2-fold undersampling, SPIRiT achieved signal-to-error ratios higher than $10 \mathrm{~dB}$ for all the evaluated voxels, except for those associated with the vertebral arteries. Note that these arteries are located in a region where the coil sensitivities are low (see Figure 1), i.e. a region with lower signal (and, therefore, lower SNR). This may have affected the performance of parallel imaging acceleration, which generally further degrades the SER. Interestingly, SoS reconstruction presents higher SER values than SPIRiT in 4 out of the 7 selected voxels. This is because, with SoS reconstruction, we observed aliasing artifacts only for the $v=0 \mathrm{~cm} / \mathrm{s}$ line. SPIRiT removes these artifacts, but introduces artifacts at other velocity components. At 4fold acceleration, aliasing artifacts are considerably stronger. Under these conditions, SPIRiT presents higher SER values than SoS reconstruction in 6 out of the 7 selected voxels, with at least $5.7 \mathrm{~dB}$ improvement for 4 of these voxels.

TABLE I

SIGNAL-TO-ERROR RATIO (IN DB) FOR 2-FOLD AND 4-FOLD ACCELERATED RESULTS, RELATIVE TO THE FULLY-SAMPLED REFERENCE.

\begin{tabular}{r|rr|rr}
\hline acceleration factor & 2-fold & & 4-fold & \\
\hline reconstruction technique & SoS & SPIRiT & SoS & SPIRiT \\
\hline spatial image & 5.03 & 16.7 & -1.3 & 9.6 \\
left carotid bifurcation & 9.0 & 11.6 & 6.1 & 8.7 \\
left jugular vein & 11.3 & 12.0 & 4.3 & 10 \\
right external carotid artery & 11.2 & 10.8 & 5.8 & 7.7 \\
right internal carotid artery & 15.1 & 11.4 & 3.0 & 9.3 \\
right jugular vein & 17.5 & 13.0 & 7.6 & 5.8 \\
right vertebral artery & 8.0 & 9.9 & 1.8 & 10.1 \\
left vertebral artery & 16.5 & 7.8 & -2.0 & 11.8 \\
\hline
\end{tabular}

\section{Discussion}

The SPIRiT results are similar to the sum-of-squares results for 2-fold acceleration, and consistently better for 4-fold acceleration. At 2-fold acceleration, SPIRiT results are very consistent and qualitatively good, relative to the fully sampled data. At 4-fold acceleration, SPIRiT results are relatively good, especially when compared with the SoS reconstruction results. This demonstrates that parallel imaging can be used in flow distribution studies in order to accelerate data acquisition and reduce scan time.

SPIRiT is able to reduce aliasing artifacts, therefore removing false signal at $v=0 \mathrm{~cm} / \mathrm{s}$. However, as it produces other types of artifacts, further analysis is required in order to validate its use for spiral FVE acceleration.

\section{CONCLUSION}

We have demonstrated parallel imaging acceleration of spiral FVE using the SPIRiT technique. The results showed that SPIRiT is able to considerably reduce spatial aliasing from the undersampled time-velocity distributions, and presents good quantitative and qualitative results for 2fold undersampling, and moderately good results for 4-fold undersampling.

For future works, we propose several aspects that can be further investigated: (i) reconstruct data from all coils, but combine the images only from those coils that are near the vessel of interest; (ii) evaluate different techniques for combining the phase images from multiple coils; (iii) evaluate other view-ordering approaches; (iv) evaluate other parallel imaging techniques, namely SENSE, CG-SENSE, TSENSE, 


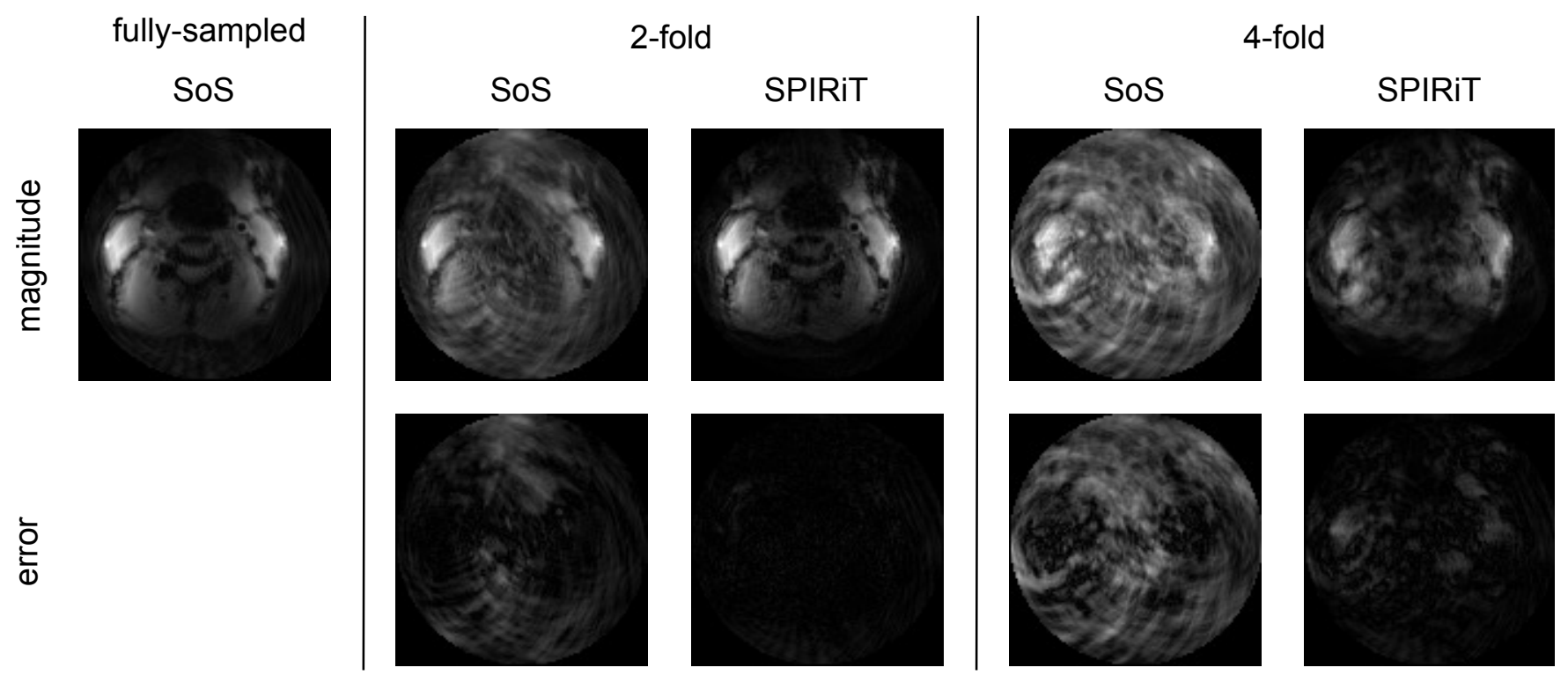

Fig. 1. Magnitude axial images of the neck (top row) obtained using sum-of-squares and SPIRiT, with different acceleration factors (fully-sampled, 2-fold and 4-fold). Reconstruction error images for both SoS and SPIRiT are also shown (bottom row). These images were reconstructed from $M\left(k_{x}, k_{y}, k_{v}, t\right)$ for $v=0$ and $t=0$.

GRAPPA, and T-GRAPPA; (v) use fast CUDA-based implementations of the reconstruction algorithms; and (vi) use parallel imaging to reduce spatial aliasing in temporallyaccelerated spiral FVE [7]. The later will enable the use of a less-selective UNFOLD filter in temporally-accelerated spiral FVE, which will improve temporal resolution for high velocities, and will make the technique more general.

\section{ACKNOWLEDGMENT}

This work was funded by a grant from the National Council for Technological and Scientific Development (Edital $\mathrm{MCT} / \mathrm{CNPq} \mathrm{N}^{\circ}$ 014/2010 - Universal). Lyra-Leite received a ProIC/DPP/UnB scientific initiation fellowship from the Brazilian National Council for Technological and Scientific Development $(\mathrm{CNPq})$. Imaging was performed at the University of Southern California, in collaboration with Prof. Krishna S. Nayak. The authors thank Kyunghyun Sung for very useful discussions.

\section{REFERENCES}

[1] Larkman, D. J. and Nunes, R. G. (2007), Parallel magnetic resonance imaging, Physics in Medicine and Biology 52:R15-R55.
[2] Moran, P. R. (1983), A flow velocity zeugmatographic interlace for NMR imaging in humans, Magn Reson Imaging 1(4):197-203.

[3] Carvalho, J. L. A and Nayak, K. S. (2007), Rapid quantitation of cardiovascular flow using slice-selective Fourier velocity encoding with spiral readouts, Magnetic Resonance in Medicine 57(4):227-288.

[4] Steeden, J. A., Jones, A., Pandya, B., Atkinson, D., Taylor, A.M. and Muthurangu V. (2012), High-resolution slice-selective Fourier velocity encoding in congenital heart disease using spiral SENSE with velocity unwrap, Magnetic Resonance in Medicine 67(6):1538-1546.

[5] Tang, C., Blatter, D. D. and Parker, D. L. (1993), Accuracy of phasecontrast flow measurements in the presence of partial-volume effects, Journal of Magnetic Resonance Imaging 3(3): 377-385.

[6] Carvalho, J. L. A., Nielsen, J. F. and Nayak, K. S. (2010), Feasibility of in vivo measurement of carotid wall shear rate using spiral Fourier velocity encoded MRI, Magnetic Resonance in Medicine 63(3):5371547.

[7] Carvalho, J. L. A and Nayak, K. S. (2007), Accelerated spiral Fourier velocity encoded imaging, Proc, ISMRM, 15th Annual Meeting, Berlin, p. 588.

[8] Lustig, M. and Pauly, J. M. (2010), SPIRiT: Iterative self-consistent parallel imaging reconstruction from arbitrary k-space, Magnetic Resonance in Medicine 64(2):457-471.

[9] Fessler, J. A. and Sutton, B. P. (2003), Nonuniform fast Fourier transforms using min-max interpolation, In: IEEE Transactions on Signal Processing 51:560-574.

[10] Roemer P. B., Edelstein W. A., Hayes C. E., Souza S. P. and Mueller O. M. (1990), The NMR phased array Magnetic Resonance in Medicine 16:192-225. 

(a)
(b)

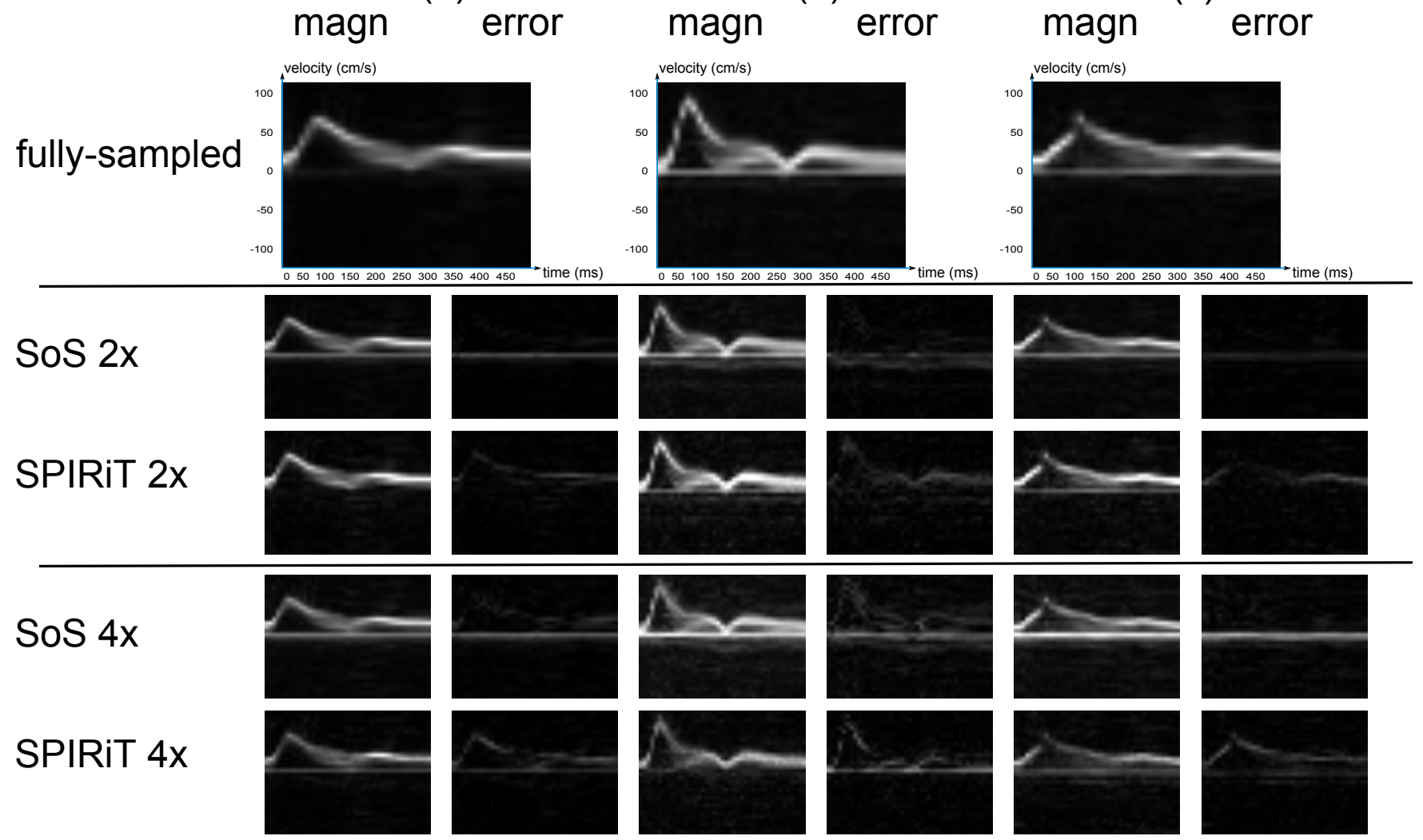

Fig. 2. Time-velocity distributions from select voxels, reconstructed using 2-fold and 4-fold sum-of-squares, and 2-fold and 4-fold accelerated SPIRiT, in comparison with the fully-sampled reference (top row) for: (a) left carotid bifurcation; (b) right external carotid artery; and (c) right internal carotid artery.

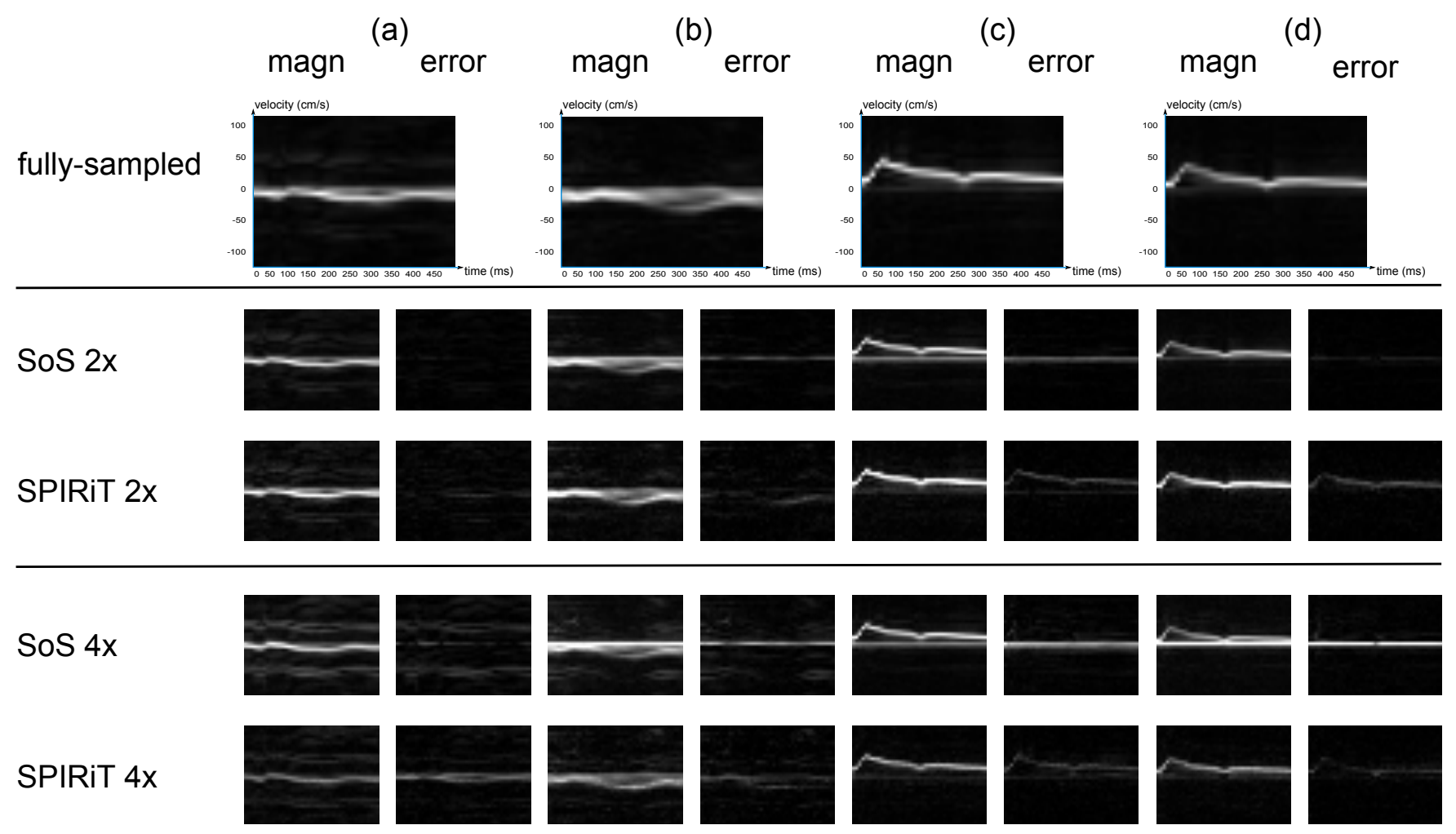

Fig. 3. Time-velocity distributions from select voxels, reconstructed using 2-fold and 4-fold sum-of-squares, and 2-fold and 4-fold accelerated SPIRiT, in comparison with the fully-sampled reference (top row) for: (a) right jugular vein; (b) left jugular vein; (c) right vertebral artery; and (d) left vertebral artery. 